\title{
A DMRG study of the $q$-symmetric Heisenberg chain
}

\author{
M. Kaulke ${ }^{\mathrm{a}}$ and I. Peschel ${ }^{\mathrm{b}}$ \\ Fachbereich Physik, Freie Universität Berlin, Arnimallee 14, D-14195 Berlin, Germany
}

July 5, 2021

\begin{abstract}
The spin one-half Heisenberg chain with $U_{q}[S U(2)]$ symmetry is studied via density-matrix renormalization. Ground-state energy and $q$-symmetric correlation functions are calculated for the nonhermitian case $q=\exp (i \pi /(r+1))$ with integer $r$. This gives bulk and surface exponents for (para)fermionic correlations in the related Ising and Potts models. The case of real $q$ corresponding to a diffusion problem is treated analytically.
\end{abstract}

\section{Introduction}

The density-matrix renormalization method (DMRG) introduced by White [1] has opened new possibilities in the study of one-dimensional quantum systems. With this approach not only the energies of large systems can be calculated very accurately, the method also yields correlation functions. [2 It was tested e.g. on Heisenberg [1] and transverse Ising [3] chains and has been applied to a variety of systems like higher-spin chains [4], ladders [5], Hubbard chains [6], impurity models [7], phonon systems [8] or transfer matrices [9,10]. It is therefore natural to try it in still other situations and to see how it performs there.

The system which we have studied is the $U_{g}[S U(2)]$ symmetric Heisenberg chain with Hamiltonian [1]

$$
H=\mp \sum_{i=1}^{L-1} e_{i}
$$

' with open boundary conditions and

$$
\begin{aligned}
e_{i}=-\frac{1}{2}\left(\sigma_{i}^{x} \sigma_{i+1}^{x}+\sigma_{i}^{y} \sigma_{i+1}^{y}\right. & +\frac{q+q^{-1}}{2}\left(\sigma_{i}^{z} \sigma_{i+1}^{z}-1\right) \\
& \left.+\frac{q-q^{-1}}{2}\left(\sigma_{i}^{z}-\sigma_{i+1}^{z}\right)\right)
\end{aligned}
$$

where the $\sigma_{i}^{\alpha}$ are Pauli matrices. Some basic features of the $q$-symmetry are sketched briefly in an appendix.

The motivation for considering this problem was twofold. Firstly, the model comprises various other physical systems and situations which thereby can be treated in a unified way. In particular, we were interested in calculating correlation functions and their exponents. Secondly, the Hamiltonian is non-hermitian for complex $q$ and offers a possibility to investigate this situation.

\footnotetext{
a e-mail: kaulke@physik.fu-berlin.de

b e-mail: peschel@aster.physik.fu-berlin.de
}

In the antiferromagnetic case $(\operatorname{sign}-)$ and for complex $q=\exp (i \pi /(r+1)), r$ integer, the chain is related to the critical models with central charge $c=1-6 /(r(r+1))$, in particular the Ising $(r=3)$ and the three-state Potts model $(r=5)$ [12, 13]. For certain real values of $q$ it corresponds to Potts models with more than three states [14]. The ferromagnetic chain with real $q$, on the other hand, describes a non-equilibrium problem, namely the hopping of classical hard-core particles on discrete sites, with a bias in one direction [15]. For $q=1$, finally, one recovers the simple isotropic Heisenberg model.

The Hamiltonian (1) has real eigenvalues and can be treated by Bethe ansatz. In this way, finite-size spectra have been obtained in connection with conformal invariance 11]. Using the Temperley-Lieb properties of the operators $e_{i}$, one can also find a representation by hermitian IRF operators, for which some DMRG calculations have already been done [16]. However, as mentioned above, we are interested in non-hermitian effects. These can be varied in this model by changing the parameter $q$.

The general arguments for the DMRG procedure [1] do not involve the Hamiltonian and the approach is therefore applicable here. In fact, non-hermitian operators have been treated occasionally 10,17,18. There is some freedom, however, which density matrix to choose then. In our case we always used a hermitian one, constructed from the right eigenvectors of (11). One then finds that its spectrum shows a marked and systematic variation with $r$. The eigenvalues decrease more slowly than for the simple Heisenberg chain. Nevertheless, there is no difficulty in obtaining the ground-state energy of $H$ to high accuracy. This is discussed in section 2 .

Our main effort involved correlations. The functions which we studied were $q$-symmetric generalizations of the quantity $\left\langle\boldsymbol{\sigma}_{l} \boldsymbol{\sigma}_{m}\right\rangle$ and correspond to fermionic and parafermionic correlators in the Ising and Potts case, respectively 19. In a previous study with exact diagonalizations of short systems no conclusive results could be obtained for the Potts model 20]. From our calculations up to 100 
sites we were able to obtain functions with clear scaling behaviour from which bulk and surface exponents could be extracted. The results are presented in section 3. In the Ising case, where one has the exact solution as a test, they are quite good. In the Potts case, there are still some uncertainties, since the bulk exponents of the four functions which we considered differ while their spinor character suggests that they are equal.

The diffusion problem, treated in section 1 , has a very different but also interesting nature. Here one finds that the eigenvalues of the density matrix drop to exactly zero very rapidly, so that the DMRG procedure terminates after a certain number of states. This can be understood from the nature of the ground state which is known here. From it, the density matrix can be derived and thus one has a non-trivial example of a system where the DMRG procedure can be carried out analytically.

A summary and some additional remarks can be found in the concluding section 5 .

\section{DMRG procedure and ground-state energies}

In the DMRG approach, a selection of relevant states is made, not for isolated, but for already interacting blocks which form the total system. In our calculations we always used the infinite-size variant of the algorithm [1].

As usual the chain was divided in two parts labeled by the indices 1 and 2, respectively. Then the right groundstate vector $\left|\Phi_{r}\right\rangle=\sum \Phi_{i j}\left|i>_{1}\right| j>_{2}$, where $\mid i>_{1}$ and $\mid j>_{2}$ denote the basis states in the two parts, and the corresponding eigenvalue $E$ were calculated by using a combination of a vector-iteration procedure (power method) and a Lanczos procedure with modified scalar product. The whole calculation was done in the $S^{z}=0$ subspace which reduced the numerical effort significantly.

From the ground-state vector the hermitian density matrix

$$
\rho=\left|\Phi_{r}><\Phi_{r}\right|
$$

of the chain was constructed where

$$
<\Phi_{r}|=| \Phi_{r}>^{\dagger}
$$

is the usual hermitian conjugate. From (3) the reduced density matrix $\rho_{1}$ of part 1 of the chain follows as

$$
\rho_{1}=\sum_{i, i^{\prime}, j} \Phi_{i j} \Phi_{i^{\prime} j}^{*}\left|i>_{1}<i^{\prime}\right|
$$

and similarly for $\rho_{2}$. Since the boundary terms in (11) break reflection symmetry one has to work with both matrices here.

The use of hermitian density matrices is already suggested by the considerations in [1] where $\rho_{1}$ enters via an optimization procedure for a vector like $\mid \Phi_{r}>$. Nonsymmetric $\rho$ 's have been used in some cases [9, 10,18] but lead to obvious problems if their eigenvalues become complex. We tested the choice $\rho=\left|\Phi_{r}><\Phi_{l}\right|$, where $<\Phi_{l} \mid$ is the left eigenvector of $H$, on the XY-analogue of (11) but it gave unsatisfactory results, in particular large errors which did not decrease properly with $m$.

The $m$ eigenvectors $\left|\lambda_{n}>_{1},\right| \lambda_{n}>_{2}$ of $\rho_{1}$ and $\rho_{2}$ corresponding to the largest eigenvalues $\lambda_{n}$ are used as new basis states for the two parts of the chain in terms of which all necessary operators are expressed. After inserting two sites between part 1 and part 2 the procedure is restarted.

For the calculation of expectation values

$$
<\hat{O}>=<\Phi_{l}|\hat{O}| \Phi_{r}>
$$

one also needs the left eigenvector. In the present case this is just the transpose of $\left|\Phi_{r}\right\rangle$ since $H$ is complex symmetric.

For the efficiency of the DMRG procedure it is important that one can calculate accurate results with a relatively small number of states of the Hilbert space. Therefore the eigenvalues of the reduced density matrix should decay exponentially. However, in general it is not a priori clear if the spectrum exhibits such a behaviour and one has to check it numerically. Figure 1 shows such spectra for different values of the parameter $r$. One can see roughly

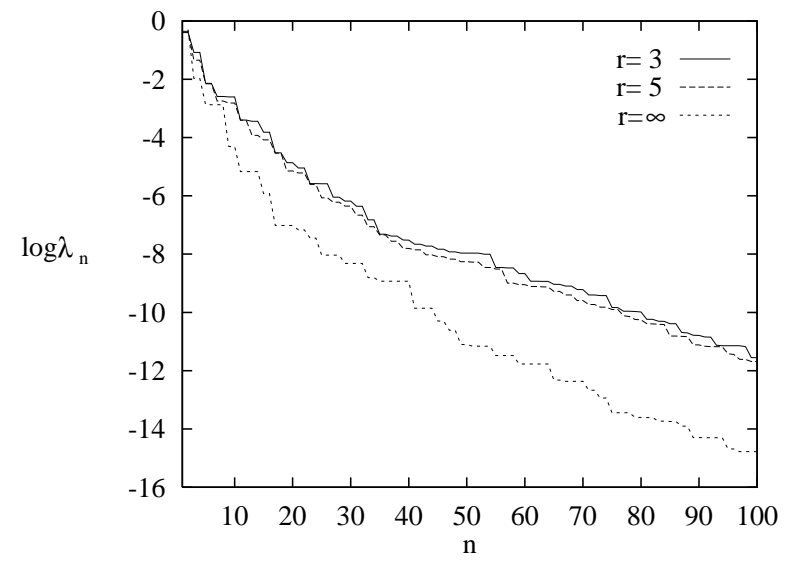

Fig. 1. Eigenvalue spectrum of the reduced density matrix $\rho_{1}$ for different values of the parameter $r$ and chain length $L=30$, calculated with 64 kept states.

exponential behaviour with a fast initial and a slower final decay. However, the results for small $r$ lie considerably above those for the isotropic Heisenberg model. The $q$ symmetric chain is therefore numerically a less favourable case. This feature is essentially due to the imaginary boundary terms. When added to an XX-chain they shift the density-matrix spectrum in the same way.

We have calculated the ground state energies for different values of the parameter $r$ and for different numbers of kept states $m$. Table 1 shows some results for $m=128$. (We note that the results given here are calculated without the constant $-(L-1)\left(q+q^{-1}\right) / 4$ in (11).) The accuracy can be checked directly for $r=3$ by comparing with the analytic result [21]. In this case the deviations are less than $10^{-9}$ which is in accord with a truncation error of the order of $10^{-9}$ in the density matrix. One can also compare 


\begin{tabular}{|c|c|c|}
\hline $\mathrm{L}$ & $\mathrm{r}=3$ & $\mathrm{r}=5$ \\
\hline \hline 4 & -0.6604497504 & -0.7393376252 \\
10 & -0.7443482698 & -0.8012448364 \\
20 & -0.7736274980 & -0.8237779981 \\
40 & -0.7885618957 & -0.8354921842 \\
60 & -0.7935870982 & -0.8394707437 \\
80 & -0.7961088422 & -0.8414746652 \\
\hline \hline $\mathrm{L}$ & $\mathrm{r}=7$ & $\mathrm{r}=\infty$ \\
\hline \hline 4 & -0.7687664240 & -0.8080127018 \\
10 & -0.8227289432 & -0.8516070414 \\
20 & -0.8427349544 & -0.8682473334 \\
40 & -0.8532195604 & -0.8770736642 \\
60 & -0.8567946053 & -0.8801004992 \\
80 & -0.8585981414 & -0.8816309100 \\
\hline
\end{tabular}

Table 1. Ground state energies per spin for different $r$, calculated with $m=128$ for $r=3,5,7$ and $m=64$ for $r=\infty$.

with the values obtained by Sierra et al. [16] in a different DMRG calculation with $m=160$. We could reproduce their data up to 9 digits.

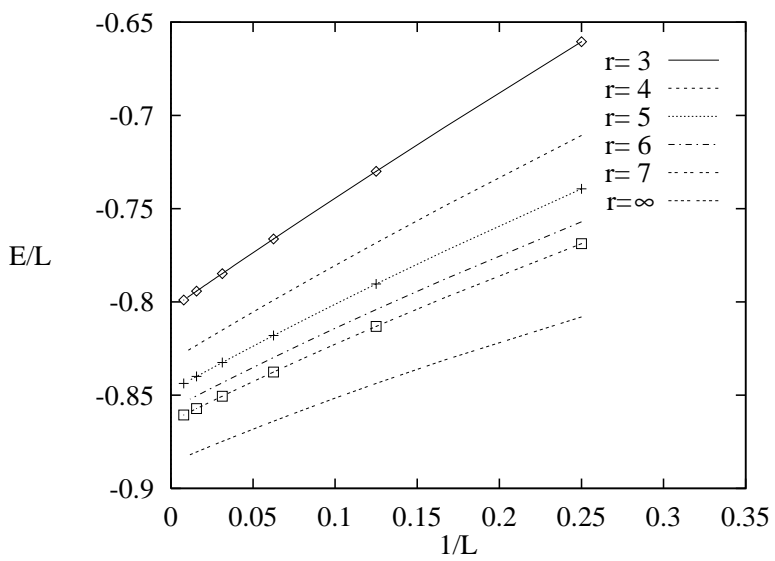

Fig. 2. Ground state energies per site for different values of $r$ calculated with $m=128$ (for $r=\infty$ with $m=64$ ). The symbols denote data from Sierra et al. 16.

Plotting the ground-state energies per spin vs. $1 / L$, one obtains the well-known asymptotic behaviour

$$
\frac{E}{L}=\epsilon_{\infty}+\frac{B}{L}-\frac{C}{L^{2}}
$$

where $B$ is the boundary energy and $C$ measures the Casimir effect. This is shown in figure 2 for several values of the parameter $r$.

One expects that for a non-hermitian operator the procedure does not necessarily give an upper bond for $E$. This is in fact the case. For $r=3$, the deviation from the exact result, although quite small, changes sign for a certain $L$. Similarly, the approach of $E$ towards its finite-size limit as a function of $m$ is not monotonous. This is shown in figure 3. One notes, however, that the behaviour becomes monotonous for large $r$.

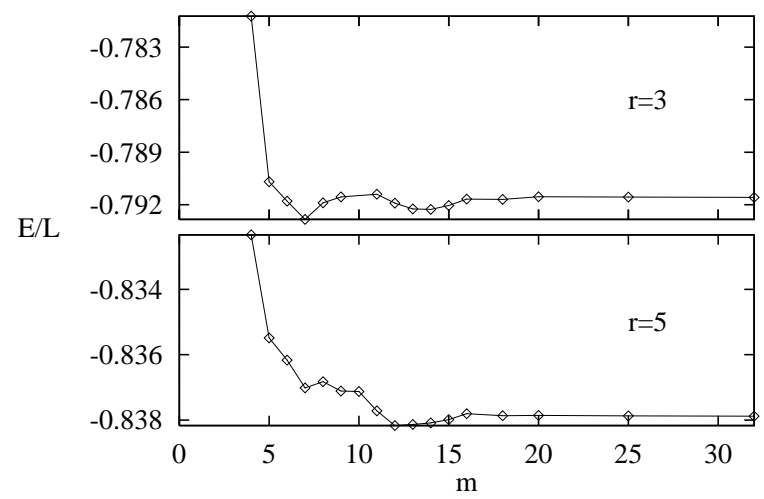

Fig. 3. Ground-state energies per site for $r=3$ and $r=5$ vs. the number of kept states $m$ of the density matrix for chain length $L=50$.

\section{Correlation functions}

For the $q$-symmetric chain with $q$ a root of unity, the usual spin correlation functions are not the appropriate quantities. Instead, we consider operators which are adapted to the $U_{q}[S U(2)]$ symmetry. In this way one is lead to generalizations of the scalar product $\boldsymbol{\sigma}_{l} \boldsymbol{\sigma}_{m}$ of two spin operators given by $[19$

$$
g_{l, m}^{ \pm}=c_{l, m}^{ \pm}-\frac{1}{q+q^{-1}}
$$

with

$$
\begin{aligned}
& c_{l, m}^{ \pm}=b_{m-1}^{ \pm} b_{m-2}^{ \pm} \ldots b_{l+1}^{ \pm} e_{l} b_{l+1}^{\mp} \ldots b_{m-2}^{\mp} b_{m-1}^{\mp} \\
& b_{j}^{ \pm}=q^{ \pm 1}-e_{j}
\end{aligned}
$$

where the $e_{j}$ are defined in equation (2). Written out explicitely, these are non-local objects containing strings of spin operators. However, they are constructed solely in terms of the $e_{j}$ from which the Hamiltonian (Ii) is built. Therefore they can be translated into any other representation of the Temperley-Lieb algebra which the $e_{j}$ fulfill [19]. In the cases $r=3$ and 5 such representations are given by the critical transverse Ising model and the Potts model with $L / 2$ sites and open boundary conditions. In these cases $<g_{l, m}^{+}>=<g_{l, m}^{-}>$, thus we work in the following only with one operator and drop the superscript.

In the Ising case one finds that the string operators cancel the Jordan-Wigner factors in a fermion picture and the $\left\langle g_{l, m}\right\rangle$ become simple fermionic two-point functions [19]. For example

$$
\begin{aligned}
<g_{2 j-1,2 k}>= & -\frac{1}{\sqrt{2}}(-1)^{j+k} \\
& \times\left\langle\left(d_{j}^{\dagger}+d_{j}\right)\left(d_{k}^{\dagger}-d_{k}\right)\right\rangle \\
<g_{2 j, 2 k-1}>= & -\frac{1}{\sqrt{2}}(-1)^{j+k} \\
& \times\left\langle\left(d_{j}^{\dagger}-d_{j}\right)\left(d_{k}^{\dagger}+d_{k}\right)\right\rangle
\end{aligned}
$$


where the $d_{j}, d_{j}^{\dagger}$ are fermion operators. These functions can be calculated exactly and are given as sums for finite $L$. The two quantities $\left\langle g_{2 j, 2 k}>\right.$ and $\left\langle g_{2 j-1,2 k-1}\right\rangle$ vanish identically. The other two correspond to different behaviour at the boundaries where (10) remains finite and (11) vanishes for fixed $k$ and finite chain lengths. Due to the open ends, one is dealing with surface critical behaviour here. After performing the continuum limit one can determine the critical exponents, and one finds the bulk exponent $x=1 / 2$ for both correlation functions and the surface exponents $x_{s}=1 / 2$ and $x_{s}=3 / 2$ for (10) and (11), respectively 19.

For the Ising case one can also construct the conventional spin correlation function $\left\langle\sigma_{l}^{x} \sigma_{m}^{x}\right\rangle$ from the quantities $e_{j}$ appearing in $H$, using the property $\left(\sigma^{x}\right)^{2}=1$. However, this is not possible for the Potts model. Also order parameter profiles, as determined by a direct DMRG calculation in [22], cannot be obtained in the present formulation. However, the $g$ 's are also interesting quantities since they become parafermion operators in this case [19,23. Their exponents will be studied in the following.

We considered the correlation function $\left\langle g_{i, L / 2}\right\rangle$ $(i=1, \ldots, L / 2-1)$ with one point fixed in the middle of the chain while varying the position of the other one. In this way profiles were computed for chains with up to 100 sites. The correlations were significantly less accurate than the energy, so that in general $m=200$ states were kept. This was a practical limit with respect to storage and time. A complete run for fixed $r$ took several weeks CPU time on middle-performance DEC-Alpha workstations. The amount of memory was about 250-300 MB RAM and up to $500 \mathrm{MB}$ harddisk space.

An example of the resulting correlation function is shown in figure 4 . The oscillations are due to the antiferromagnetic character of the chain. Except for the case

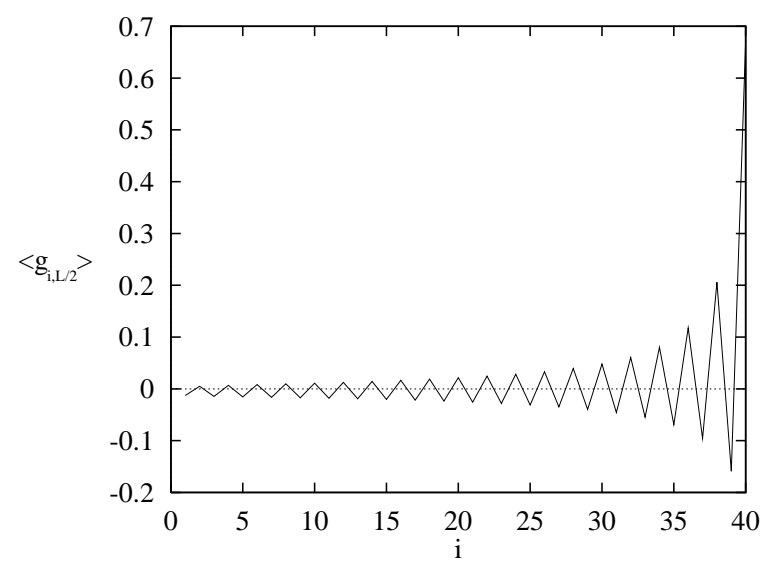

Fig. 4. Correlation function $\left\langle g_{i, L / 2}\right\rangle$ for $r=5$ and chain length $L=82$, calculated with 200 kept states.

$r=3$, where only the two functions (10) and (11) are nonzero, one can distinguish four different functions given by the maxima and minima for even and odd $L / 2$.

As a check on the accuracy, one can compare with exact results for $L=24$ [24]. One then finds deviations less than $10^{-4}$ in the Ising case and less than $10^{-5}$ in the Potts case while the truncation error is only $10^{-14}-10^{-15}$. The correlations are therefore much less precise than the ground-state energy. One also finds that the values can be above or below the exact result. The errors increase, as usual in the DMRG, as one moves towards the boundary. This effect can also be seen in figure 5, where two correla-

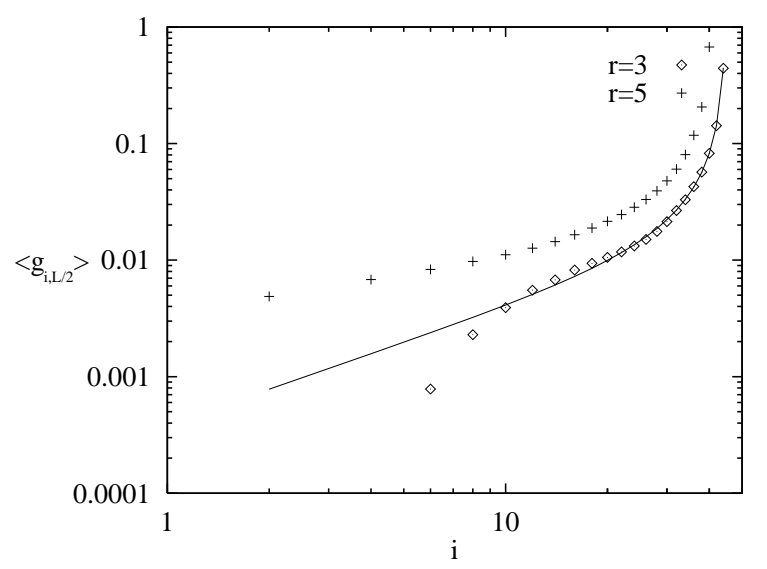

Fig. 5. Log-log plot of the correlation function $\left\langle g_{i, L / 2}\right\rangle$ for even $i$ and chain length $L=82(r=5)$ and $L=90(r=3)$, calculated with 200 kept states. The curve denotes analytic results for $r=3$.

tion functions are shown for large systems. Up to $R \simeq 30$, where $R=L / 2-i$ is the distance from the center, the numerical values for $r=3$ reproduce the analytical ones quite well. Near the boundary, where the function is very small, however, there are considerable deviations and the expected power-law behaviour $g \sim R_{s}^{x_{s}-x}$, with $R_{s}=i$ denoting the distance from the surface, breaks down. This is different for $r=5$, where the curve remains linear also in this region. One therefore expects that the results for larger $r$ are more accurate.

In the middle of the chain, the situation is more favourable since the quantities have undergone fewer iterations here. For small $R(R \ll L / 2)$ one finds the expected bulk behaviour $g \sim R^{-2 x}$, as shown in the log-log plot of figure 6. However, if one determines the exponent $x$ from this data for $r=3$, one still has a relative error of about $5 \%$.

In order to improve the accuracy, we used the scaling form for $g$

$$
g(R, L)=\frac{1}{R^{2 x}} F\left(\frac{R}{L}\right)
$$

where $F(R / L) \rightarrow$ const for $R / L \rightarrow 0$. Collecting results for different $L$ we constructed a scaling plot $R^{2 x} g$ vs. $R / L$ and varied $x$ until the data fell onto a curve as well as possible. The result of such a procedure for $r=5$ is shown in figure 7. It proves that scaling is indeed fulfilled, in contrast to [20] where it could not be seen for chains up to $L=28$. The exponent $x$ for $r=3$ then is only about $2 \%$ off the exact result $x=1 / 2$.

A further improvement could be achieved by extrapolating the bulk values of $g$ to the infinite-chain limit. Then 


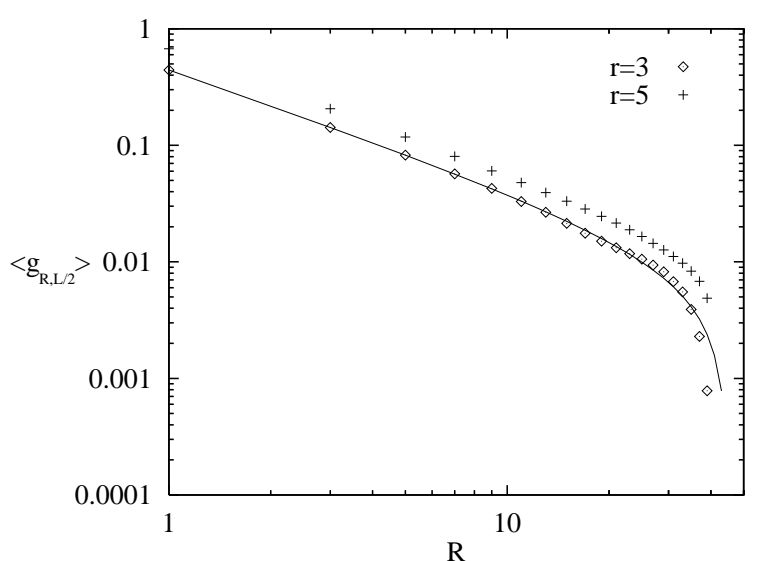

Fig. 6. Log-log plot of the correlation function $\left\langle g_{R, L / 2}\right\rangle$, $R=L / 2-i$, for odd $R$ and chain length $L=82(r=5)$ and $L=90(r=3)$, calculated with 200 kept states. The curve denotes analytic results for $r=3$.

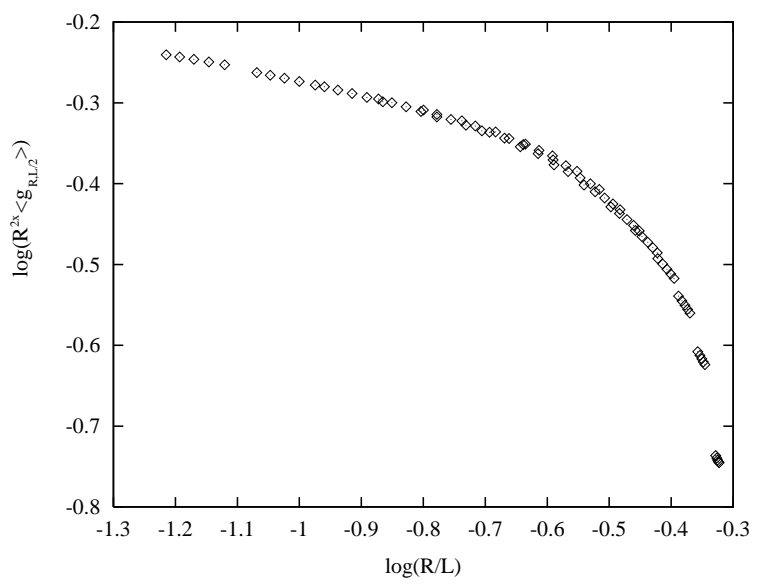

Fig. 7. Finite-size scaling behaviour of the correlation function $<g_{R, L / 2}>, R=L / 2-i$, for $r=5$ and five chain lengths from $L=66$ to $L=82$, calculated with 200 kept states.

a linear fit of the data in the corresponding plot gave better Ising exponents. In this way, the results in table 2 were determined. The confidence interval of the fit parameters would give rise to an absolute error in the exponents less than $10^{-3}$, but the error in the data points is not known. Thus we cannot give a reasonable error bar but we can use the results for $r=3$ as a clue.

\begin{tabular}{|c|c|c|c|c|}
\hline $\mathrm{r}$ & $\left\langle g_{2 j, 2 k}\right\rangle$ & $\left\langle g_{2 j, 2 k-1}\right\rangle$ & $\left\langle g_{2 j-1,2 k}\right\rangle$ & $\left\langle g_{2 j-1,2 k-1}\right\rangle$ \\
\hline \hline 3 & - & 0.499 & 0.502 & - \\
\hline 4 & 0.386 & 0.521 & 0.513 & 0.406 \\
\hline 5 & 0.397 & 0.520 & 0.508 & 0.409 \\
\hline 6 & 0.392 & 0.516 & 0.502 & 0.417 \\
\hline 7 & 0.412 & 0.499 & 0.484 & 0.422 \\
\hline
\end{tabular}

Table 2. Critical exponents $x$ of the four correlation functions, calculated with $m=200$. Exact results are known for $r=3$ and $r=\infty$ where $x=1 / 2$.
The final Ising exponents differ only by $10^{-3}$ from the exact value and thus are very good. The situation is more complicated, though, for other cases. The Kac table [25] for $r=5$ contains 10 different conformal dimensions and allows for a large number of combinations $x=\Delta+\bar{\Delta}$. However, if one fixes the spin $s$ of the operators to simple values, only $x=11 / 20(s=1 / 2), x=7 / 15(s=1 / 3)$ and $x=2 / 3(s=2 / 3)$ remain. These values were also obtained directly by relating the three-state Potts model to a Gaussian model [26]. From the explicit form of the parafermion operators (products of local and string operators) [19], it can be seen that they have spinor properties as discussed in 26] with $s=1 / 3$. Thus one would expect a common exponent $x=0.467$ for all four $g$ 's, whereas the numerical values lie about $10 \%$ higher and lower. In principle, they would allow also other $x$-values connected with strange spin. A similar situation is found for $r=4$ (tricritical Ising model) where $x=7 / 16$ (0.438), $x=19 / 40$ (0.475) and $x=43 / 80(0.537)$ are close to the measured values.

The reason for the discrepancies is not clear. As noted above, one would expect better accuracy for the Potts model. If the $x$-values actually differ, it could perhaps be due to a cancellation of leading singularities in the $g$ 's which, when written in the Potts representation, are sums of two contributions. The other possibility is that in spite of the satisfactory scaling behaviour the numerical results are not yet good enough.

We also determined the surface exponents $x_{s}$. This was done with the help of another scaling plot. For $i=R_{s}$, the scaling form of $g$ can be written as

$$
R_{s}^{2 x} g\left(R_{s}, L\right)=\left(\frac{R_{s}}{L}\right)^{x_{s}+x} G\left(\frac{R_{s}}{L}\right)
$$

where $G \rightarrow$ const for $R_{s} / L \rightarrow 0$. Using the bulk exponents as determined above, one can now find the surface exponent by tuning $x_{s}$ until the scaling behaviour (13) is fulfilled. In this procedure the first few sites near the boundary, for which the errors are large, were left out.

For the Ising case we found $x_{s}=1.58$ for the function (10) and $x_{s}=0.56$ for the function (11). One notes that these results differ more from the exact values than the bulk results. For the Potts case the following exponents were found: $x_{s}=0.81$ for $\left\langle g_{2 j, 2 k}\right\rangle, x_{s}=1.05$ for $\left\langle g_{2 j, 2 k-1}\right\rangle, x_{s}=0.63$ for $\left\langle g_{2 j-1,2 k}\right\rangle$ and $x_{s}=0.55$ for $\left\langle g_{2 j-1,2 k-1}>\right.$.

We have also determined the exponents for higher $r$. The values tend to their Heisenberg limits $x=1 / 2$ and $x_{s}=1$ for $r \rightarrow \infty$. However, while the exponents of the function $<g_{2 j, 2 k-1}>$ approach this limit from above, the exponents of the other functions always lie below it. Furthermore, the limiting values are not easy to obtain due to the logarithmic corrections which occur for $q=1$ [27.

\section{$4 q$-symmetric driven diffusion}

It is well-known that the isotropic ferromagnetic Heisenberg model also describes (in all dimensions) the hopping 
of classical particles on a lattice with the exclusion of double occupancy 28]. This follows by expressing the master equation for the probability vector $|P\rangle$ describing the system

$$
\frac{\partial}{\partial t}|P>=-H| P>
$$

in a spin one-half language where a spin up (down) corresponds to an occupied (empty) site. The stationary state is therefore the ferromagnetic ground state in the corresponding sector of fixed $S^{z}$.

A similar result holds if there is a bias in the hopping. In one dimension and after a canonical transformation the time-evolution operator then takes exactly the form (i) with $q$ given by $q=\left(\alpha_{-} / \alpha_{+}\right)^{1 / 2}$ where $\alpha_{+}\left(\alpha_{-}\right)$is the hopping rate to the right (left) [15]. The stationary properties of this diffusion problem have already been studied [29]. Due to the bias, the particles accumulate at one end, thus producing a non-trivial density profile. In the magnetic language, one is dealing with a uniaxial ferromagnet to which opposite boundary fields are applied at the two ends. These are real and $H$ is hermitian in this case.

The numerical treatment leads to a density-matrix spectrum as shown in figure 8 . The eigenvalues decrease faster than exponentially and become effectively zero be-

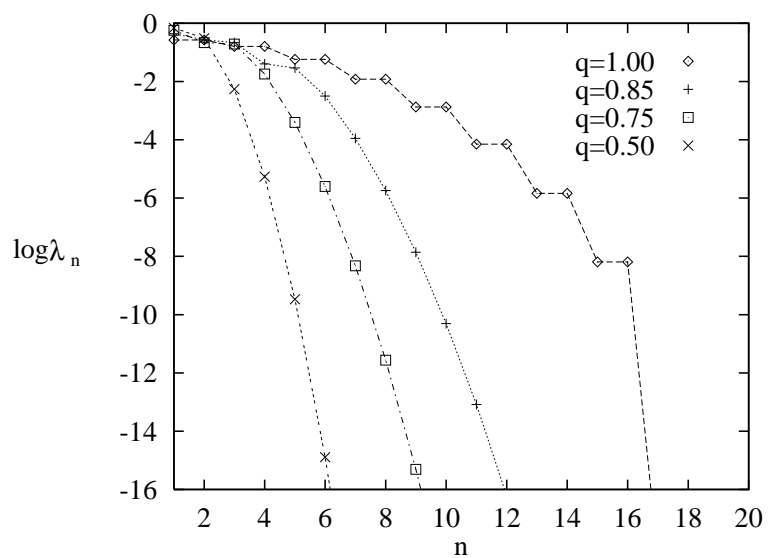

Fig. 8. Eigenvalue spectrum of the reduced density matrix $\rho_{1}$ of the $q$-symmetric hopping model at half-filling for different values of the parameter $q$, calculated with 16 kept states and chain length $L=30$.

yond a certain point depending on the size of the system. The decrease also depends on the asymmetry parameter $q$.

To understand this, it is useful to consider the case $q=1$ first. Then the ground state of the chain is a spin state $\mid S, M>$ where $S=L / 2$ and $M=S^{z}$ can be chosen. Taking $M=0$ and dividing the chain into two halves, it can be written as

$$
|\Phi>=| S, 0>=\sum_{m=-s}^{+s} c_{m}\left|s, m>_{1}\right| s,-m>_{2}
$$

where $\mid s, m>_{1,2}$ are the corresponding spin states of the two subsystems with $s=S / 2=L / 4$ and the $c_{m}$ are the appropriate Clebsch-Gordan coefficients. Thus $\mid \Phi>$ is the superposition of $(S+1)$ terms and the density matrix $\rho_{1}$ of part 1 of the chain has only that many non-zero eigenvalues. It reads explicitely

$$
\rho_{1}=\sum_{m}\left|s, m>_{1} c_{m}^{2}{ }_{1}<s, m\right|
$$

so that these eigenvalues are given by $c_{m}^{2}$.

For $q \neq 1$ the situation does not change, because there are analogous decomposition formulae for the $q$-deformed angular-momentum states [30]. In this case one finds

$$
c_{m}=\frac{1}{\sqrt{[4 s] !}} \frac{[2 s] !^{2}}{[s-m] ![s+m] !} q^{2 s m}
$$

with the notation

$$
[n]=\frac{q^{n}-q^{-n}}{q-q^{-1}}
$$

and

$$
[n] !=[1] \cdot[2] \cdot \ldots \cdot[n] .
$$

For $q \rightarrow 1,[n]$ reduces to $n$ and (17) becomes the normal Clebsch-Gordan coefficient. One can check that the $c_{m}^{2}$ given by (17) are precisely the density-matrix eigenvalues obtained in the numerical procedure.

Due to the $q$-factor in (17) there is no symmetry between $m$ and $-m$ for $q \neq 1$ and the degeneracy of the eigenvalues is lifted as seen in figure 8. Specifically for large $q$ one has

$$
c_{m} \simeq q^{-(s-m)^{2}}
$$

so that the coefficient $c_{s} \simeq 1$ dominates and $\mid \Phi>$ becomes approximately a product state

$$
|\Phi>\simeq| s, s>_{1} \mid s,-s>_{2} .
$$

Physically this means that one half of the chain is almost filled with particles and the other one is almost empty, as expected for large bias. Expressions for the density profile were given in 29 and are easily reproduced by the DMRG calculations. Similarly, the treatment can be extended to $M \neq 0$, i.e. to systems which are not half-filled. Then the number of terms in $\mid \Phi>$ is even smaller, namely $(S+$ $1-|M|)$, and becomes one for a completely full or empty system. The DMRG procedure gives exact results once $m$ exceeds this value which increases at most linearly with the system size.

\section{Conclusion}

We have studied the $q$-symmetric Heisenberg chain for various cases of physical interest. For the case of complex $q$ we showed that the DMRG procedure works well even though the density-matrix spectra are less favourable due to the non-hermitian boundary terms. We found the ground-state energies to high accuracy and calculated also generalized correlation functions, for which we determined the critical exponents. 
The study of various different models via the spin onehalf operator $H$ was also motivated numerically, since the dimension of the matrices in the DMRG procedure is lower then. The results show, however, that to some extent this advantage is compensated by the sensivity of the correlations in this formulation. One should also note that the equivalent Potts chain has only half the length of the $q$ symmetric chain. Thus it seems that one cannot really circumvent certain features of the corresponding problems. To improve the results further, one would have to increase the numerical effort, by using larger values of $m$ or the sweeping procedures in the finite-size algorithm [1.

The ferromagnetic chain for real $q$ was seen to have very different features and is interesting in other respects. Firstly, as an example where the density matrix can be found analytically and the DMRG procedure automatically gives the exact result. There are only a few other cases of this kind, for example two coupled oscillators [31] or finite-dimensional matrix-product states [32]. Secondly, it describes not only a magnetic problem, but also a nonequilibrium system. The use of the DMRG in this field, where the time evolution operators are in general nonhermitian, has only started [18 and further interesting applications can be expected.

\section{Appendix}

The $q$ - or quantum group symmetry (see e.g. 13]) is a generalization of rotational symmetry and characterized by a modified commutator

$$
\left[S^{+}, S^{-}\right]=\left[2 S^{z}\right]
$$

between the generators. Here the bracket is defined in (18) so that a $q$-dependent function of $S^{z}$ appears on the right. For $q=1$, the usual angular momentum algebra is recovered. For a chain of spins, $S^{z}=\sum \sigma_{n}^{z} / 2$ has the usual form, but $S^{ \pm}$are given by

$$
S^{ \pm}=\frac{1}{2} \sum_{n=1}^{L} q^{\sum_{l=1}^{n-1} \sigma_{l}^{z} / 2} \sigma_{n}^{ \pm} q^{-\sum_{l=n+1}^{L} \sigma_{l}^{z} / 2} .
$$

The property $\left[H, S^{ \pm}\right]=0$ of the Hamiltonian (1) can be used, as in the case $q=1$, to obtain all ferromagnetic ground states from the one with all spins up via repeated application of $S^{-}$29. In this way one can also derive the Clebsch-Gordan coefficients (17).

\section{Acknowledgments}

We would like to thank H. Hinrichsen, V. Rittenberg, F. Iglói, M. Henkel and H. Niggemann for useful discussions and P. Arndt and T. Heinzel for correspondence. We furthermore thank X. Wang for his advice in the DMRG method, A. Honecker for help with the numerics, the Université Henri Poincaré, Nancy, for hospitality and the Max-Planck-Institut für Physik komplexer Systeme in Dresden for substantial computer time.

\section{References}

1. S.R. White, Phys. Rev. Lett. 69, (1992) 2863; S.R. White, Phys. Rev. B 48, (1993) 10345.

2. For a short review see G.A. Gehring, R.J. Bursill and T. Xiang, Acta Physica Polonica A 91, (1997) 105.

3. Ö. Legeza and G. Fáth, Phys. Rev. B 53, (1996) 14349.

4. U. Schollwöck and T. Jolicoeur, Europhys. Lett. 30, (1995) 493; K. Hallberg, X.Q.G. Wang, P. Horsch and A. Moreo, Phys. Rev. Lett. 76, (1996) 4955.

5. S.R. White, R.M. Noack and D.J. Scalapino, Phys. Rev. Lett. 73, (1994) 886; K. Hida J. Phys. Soc. Jap. 64, (1995) 4896; S.R. White and D.J. Scalapino Phys. Rev. B 55, (1997) R14701; B 57, (1998) 3031.

6. S. Qin, S. Liang, Z. Su and L. Yu, Phys. Rev. B 52, (1995) R5475.

7. X. Wang and S. Mallwitz, Phys. Rev. B 53, (1995) R492; X. Wang, cond-mat/9705302, (1997).

8. L.G. Caron and S. Moukouri, Phys. Rev. Lett. 76, (1996) 4050; Phys. Rev. B 56, (1997) R8471.

9. T. Nishino, J. Phys. Soc. Jap. 64, (1995) 3598; T. Nishino and K. Okunishi, J. Phys. Soc. Jap. 66, (1997) 3040.

10. R.J. Bursill, T. Xiang and G.A. Gehring, J. Phys.: Cond. Mat. 40, (1996) L583; X. Wang and T. Xiang, Phys. Rev. B 56, (1997) 5061; N. Shibata, J. Phys. Soc. Jap. 66, (1997) 2221.

11. F.C. Alcaraz, M.N. Barber and M.T. Batchelor, Phys. Rev. Lett. 58, (1987) 771; F.C. Alcaraz, M.N. Barber, M.T. Batchelor, R.J. Baxter and G.R.W. Quispel, J. Phys. A: Math. Gen. 20, (1987) 6397.

12. F.C. Alcaraz, M. Baake, U. Grimm and V. Rittenberg, J. Phys. A 22, (1989) L5.

13. V. Pasquier and H. Saleur, Nucl. Phys. B 330, (1990) 523.

14. F.C. Alcaraz, M.N. Barber and M.T. Batchelor, Ann. Phys. 182, (1988) 280.

15. F.C. Alcaraz, M. Droz, M. Henkel and V. Rittenberg, Ann. Phys. 230, (1994) 250.

16. G. Sierra and T. Nishino, Nucl. Phys. B 495, (1997) 505.

17. J. Kondev and J.B. Marston, Nucl. Phys. B 497, (1997) 639.

18. Y. Hieida, J. Phys. Soc. Jap. 67, (1998) 369.

19. H. Hinrichsen, P.P. Martin, V. Rittenberg and M. Scheunert, Nucl. Phys. B 415, (1994) 533.

20. P.F. Arndt and T. Heinzel, J. Phys. A: Math. Gen. 28, (1995) 3567.

21. T.W. Burkhardt and I. Guim, J. Phys. A: Math Gen. 18, (1985) L33.

22. E. Carlon and F. Iglói, cond-mat/9710144, (1997).

23. L. Mittag and M.J. Stephen, J. Math. Phys. 12, (1971) 441.

24. T. Heinzel, Diplomarbeit (Bonn 1995).

25. P. Christe and M. Henkel, Introduction to Conformal Invariance and Its Applications to Critical Phenomena (Springer, Berlin 1993).

26. B. Nienhuis and H.J.F. Knops, Phys. Rev. B 32, (1992) 1872.

27. K.A. Hallberg, P. Horsch and G. Martínez, Phys. Rev. B 52, (1995) R719.

28. S. Alexander and T. Holstein, Phys. Rev. B 18, (1978) 301; W. Dieterich, P. Fulde and I. Peschel, Adv. in Physics 29, (1980) 527.

29. S. Sandow and G. Schütz, Europhys. Lett. 26, (1994) 7. 
30. A. Kirillov and N.Y. Reshetikhin in Infinite Dimensional Lie Algebras and Groups ed. V.G. Kac (World Scientific, Singapore 1989).

31. D. Han, Y.S. Kim and M.E. Noz, cond-mat/9705029, (1997).

32. see, e.g. A. Klümper, A. Schadschneider and J. Zittartz, Europhys. Lett. 24, (1993) 293; F. Eßler and V. Rittenberg, J. Phys. A: Math. Gen. 29, (1996) 3375. 